\title{
El concepto de persona, como determinante del cuidado
}

\author{
The person concept; like determinant \\ of the care
}

\author{
Lic. Enf. y Obst. Laura Jiménez Trujano*, Mtra. Rosa María Ostiguín Meléndez ${ }^{\star \star}$, Mtro. Alfredo Bermú- \\ dez González ${ }^{* * *}$ \\ * Alumna de la $5^{a}$ generación del Programa de Maestría en Enfermería (PME), ${ }^{\star \star}$ Profesora Titular A. \\ Coordinadora de la Maestría en Enfermería, ENEO - UNAM, ${ }^{* * *}$ Profesor adscrito a la Unidad de Inves- \\ tigación y posgrado de la ENEO-UNAM.
}

\section{Resumen}

Al revisar la evolución del conocimiento enfermero, Fawcet, identifica como componentes coincidentes citados por las teóricas 4 elementos que forman el metaparadigma constituido por; persona, cuidado, salud y entorno, cabe hacer notar que el concepto de cada uno de ellos, esta influenciado por la visión de la teórica que lo ha definido y por la corriente de pensamiento de la que surge.

Para ello se revisa la evolución que el concepto de persona ha tenido en México, influido por los antecedentes prehispánicos, la herencia vocacional religiosa y el desarrollo del concepto en el cuidado contemporáneo, a partir de la educación formal de las enfermeras en los hospitales a finales del siglo XIX, principios del XX, lo que tiene un impacto determinante en la visión de cuidado y persona,

In a constant review of the evolution of nursing knowledge, Fawcet identifies four balanced components quoted by theory 4 elements as part of the met paradigm, which are: Person, Care, Health and Surroundings. It's important to notice that the meaning of each of the concepts mentioned above is influenced by the vision of the theory that is has defined it and from the source these concepts come from.

That is why the evolution of the concept of person is reviewed constantly in Mexico, influenced as well by its prehispanic background, religious heritage and the development of the concept as far as contemporary care is concerned since the formal education of nurses took place in hospitals at the end of the XIX century, the beginning of the XX century. This evolution has a determinant impact in the vision of the ya que centra la atención en la enfermedad, lo biológico y la técnica, enfoque que ha predominado hasta fechas recientes.

Recientemente existe interés en comprender al hombre en su dimensión de individuo, en percibirlo como tal y trascender a la humanización del cuidado, para ello las teóricas han incursionado en otras áreas del conocimiento. La propuesta de éste ensayo es profundizar desde la filosofía el concepto de persona, para entender que se debe constituir la atención para cada sujeto, para ayudar a su trascendencia como tal.

Palabras clave: Persona, cuidado, evolución, humanización.

\section{Abstract}

care and person, which focuses on the aliment, the biological field and the skill, approach that has been determinant up to the present.

Recently, there exist an interest in understanding the concept of man in its individual dimension, in understanding him as such, and transcend it to the humanization of the care, in which quite a few new techniques have been developed and integrated in other areas of knowledge. The proposal of this essay is to get into these concepts in a deeper way from the philosophy of the concept of person, to understand that all the attention should be drawn to the individual, to help him go beyond his expectancies.

Key words: Person, Care, Evolution, Humanization 


\section{INTRODUCCIÓN}

Cuando se revisa la evolución del conocimiento enfermero, sobresalen las enfermeras teóricas para quienes fue evidente la necesidad de desarrollar, articular, así como probar el conocimiento teórico de Enfermería, en la búsqueda de reconocimiento como disciplina académica, lo que ocurre a partir de la década de los 50's. Fawcett es quien identifica 4 elementos presentes que coinciden en los escritos de las teóricas, estos son los llamados elementos del metaparadigma: Persona, cuidado, salud, entorno'; sin embargo el concepto de cada uno de estos esta influenciado por la visión de la teórica que lo ha trabajado, así como de la corriente de pensamiento del que surge, encontrando diversidad en su conceptualización.

Comprender que el cuidado proporcionado por enfermería, es determinado por todo lo que conforma al sujeto que valora y que tiene un significado para el depositario de la atención ${ }^{2}$ hace necesario analizar el cómo observar a la persona asistida, ya que ello determina la vigilancia que brinda la Enfermera y permite identificar si existe evolución en el conocimiento de la disciplina. Para ello es importante indagar la visión de individuo que ha predominado en diferentes épocas, para comprender el cuidado de enfermería que se ha suministrado. Es así que se aborda la conceptualización de persona, por parte de diversos pensadores desde el punto de vista filosófico, para partir hacia la reflexión del concepto de persona.

\section{El cuidado en México}

Cuidar es una actividad que ha permitido mantener la vida del ser humano, el rol de cuidadora ha sido asignado a la mujer como consecuencia del proceso de socialización familiar y de división del trabajo, en donde se le ha establecido el papel de cuidadora de la familia y criado$\mathrm{ra}^{3}$, estos son los antecedentes en la etapa llamada doméstica del cuidado. En México en la época prehispánica semejante a las sociedades occidentales, se concedía el papel de cuidadora de la familia a la mujer, relegándola a la realización de actividades domésticas ${ }^{4}$.

En la cultura azteca, un ejemplo del cuidado a la vida, lo encontramos en la importancia que daban al niño, cuando se confirmaba el embarazo, la tlamatiqui ticitl (partera $)^{5}$ era la encargada de proveer cuidados a la mujer para asegurar la salud del niño por venir, ella era quien lo recibía y daba la bienvenida con palabras de amor y ternura, llamándolo "pluma rica o piedra preciosa" ${ }^{\circ}$, al mismo tiempo que cortaba el cordón umbilical y proporcio- naba el primer baño, orando para la purificación del niño ${ }^{7}$, denotando que daban importancia a aspectos afectivos y no sólo cuidaban las necesidades físicas de las personas, al ser prácticas promovidas como grupo, formaban parte de la cultura.

Cuando ocurre la conquista se presenta el proceso de aculturación ${ }^{8}$, en donde existe una mezcla de la medicina tradicional (pueblos vencidos) con la occidental (conquistadores); sin embargo el cuidado de los enfermos queda a cargo de la iglesia; ya que se ordenaba que en todos los pueblos hubiese un hospital cerca de ella, institucionalizando así la atención de los enfermos, con lo que quedaba bajo el mando del clero como ocurría en Europa, excluyendo las prácticas ancestrales de cuidado, las cuales se consideraban hechicería y supersticiones.

Los antecedentes de la Enfermería moderna, se encuentran en el cristianismo, en esa época se ve a la persona cuidada con sentimientos caritativos y de compasión, en donde se anteponen las necesidades del alma, a la satisfacción de las necesidades básicas del enfermo (higiene, nutrición) y la aplicación de tratamientos; en esa época hay una actitud de abnegación por quienes brindan el cuidado, ya que manifiestan sumisión ante médicos y sacerdotes, de este modo, el cuidar a los enfermos se convierte en vocación ${ }^{9}$

Nightingale, es la fundadora de la enfermería moderna, tiene gran influencia del humanismo, ella se refería a la persona como paciente en donde le asigna a la enfermera un papel activo, controlando el entorno y a el individuo el papel pasivo en el cuidado, ella no define dicho elemento, sin embargo señala su interés por él, como compasión caritativa $^{10}$; aunque también considera a la persona en sus componentes físico, intelectual, emocional y espiritual $^{11}$, evidenciando con algunas características inherentes a la persona.

\section{El cuidado contemporáneo}

En México, la formación de las enfermeras, a semejanza de lo que ocurre en Europa y Estados Unidos, se realiza en los hospitales a fines del siglo XIX y principios del siglo $X X^{12}$, esto va a tener una influencia determinante en la visión del cuidado y de persona cuidada que prevalece hasta nuestros días. En el Hospital General de México el 9 de febrero 1907 inicia la formación de enfermeras por iniciativa del Dr. Fernando López y el Dr. Liceaga, en 1911 se incorpora a la Universidad Nacional de México ${ }^{13,14}$, siendo el enfoque a la enfermedad, hacia lo biológico, así como 
a los procedimientos técnicos, lo que se privilegia en la enseñanza, como ocurre en otros países.

A partir de los 60's en el Hospital General y de los 70's en el IMSS ${ }^{15}$, se da auge a la formación en los postécnicos de enfermería, semejantes a las especialidades médicas; enfermería quirúrgica, pediatra, e intensivistas en donde se continúa privilegiando el paradigma positivista enfatizando la enseñanza de lo biológico (el ser humano es una enfermedad u objeto de estudio) y destaca la preparación en el uso de la técnica y procedimental, aunque asoman algunas actividades de educación a los pacientes.

Lo anterior nos lleva a sólo ver una parte de la persona, la biológica, lo objetivo, desapareciendo lo subjetivo que influye también en la experiencia de la salud, impidiendo con ello la comunicación y la interacción con la persona enferma y su familia ${ }^{16}$, todo ello en el marco de las instituciones de salud.

Actualmente existe interés en trascender más allá de ese modelo, esto se evidencia en el interés de acercarnos al ser con el otro, es decir comprender al hombre en su dimensión humana, ello surge a través del replanteamiento del cuidar y del ser en quien "es depositado" el cuidado, ya que es evidente que la enfermedad vivenciada por una persona, no sólo afecta lo físico, sino su propia identidad ${ }^{17}$; así el cuidado es una actitud para con el otro, una relación de responsabilidad interhumana que nos permite satisfacer, conocer y compartir experiencias y comportamientos con otras personas, para promover su bienestar ${ }^{18}$.
Heidegger menciona que el ser llega a ser destino en la medida en que se da, el adueñarse de una persona en su esencia es amar, el hombre no es el dueño del ser, es sólo su cuidador; en virtud de ello, si la persona que brinda cuidado introyecta esta postura, estará en posibilidad de ser custodio del otro ser y de ese modo llegar a comprender al individuo en su totalidad, para humanizar la atención.

Es por lo anterior que las enfermeras a partir de la experiencia y la reflexión de su quehacer, dan origen a las teorizaciones sobre la disciplina, su formación en áreas como la educación, antropología, psicología, etnografía, les hacen reconocer en enfermería, una profesión diferente a la médica, en donde se motiva el surgimiento de investigaciones alrededor del objeto de cuidado, o sea la persona y su entorno ${ }^{19}$. Si bien esto ocurre en los Estados Unidos, es innegable la repercusión que en América Latina y particularmente en México han tenido estas reflexiones, aunque aun no se han generalizado en el grupo de profesionales en nuestro país.

Es importante, reconceptualizar a la persona quien es el objeto de cuidado en la disciplina de Enfermería, para ello partiremos de la definición que da Boecio, quien menciona que la persona es sustancia individual de naturaleza racional ${ }^{20}$, enfatizando en la razón como característica que permite actuar a los hombres a partir de ella y convertirlos en personas. Santo Tomás agrega; lo que separa a la persona humana de lo existente en la naturaleza es el poseer cuerpo y alma.

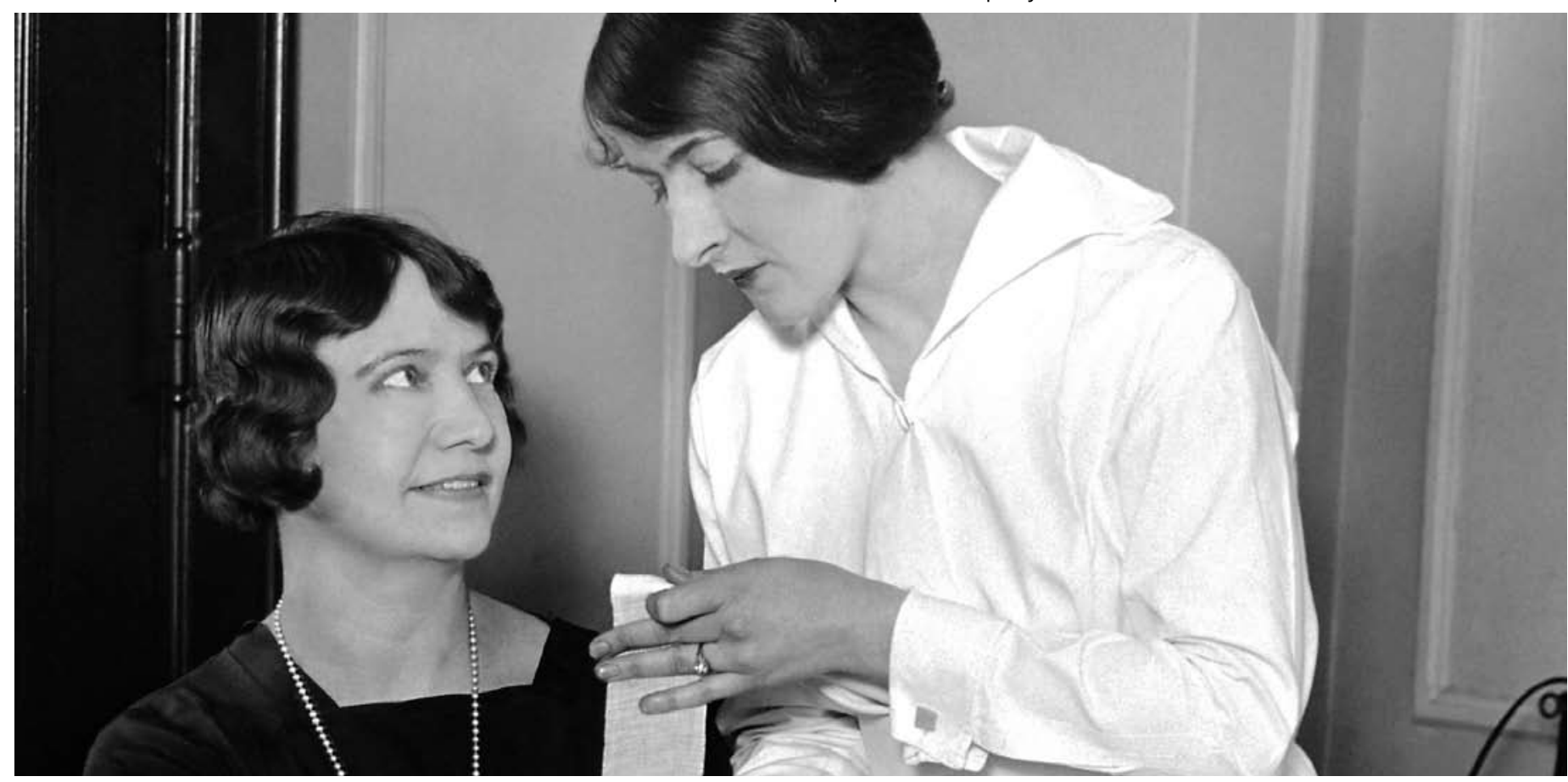


Leibniz hace hincapié en tres características del ser, la individualidad, pluralidad y causalidad ${ }^{21}$; la individualidad que distingue a uno del otro (no existen 2 seres iguales) y que sin embargo admite la pluralidad del ser, consistente en cada parte de la materia que conforma la individualidad. También posee causalidad, es decir darse cuenta de lo que se desea y ello es evidencia de la libertad; aspectos de los que actualmente se reflexiona en Enfermería para hablar de la experiencia del cuidado.

Con Locke por su parte habla de la conciencia, del ser humano con inteligencia, pensante y reflexivo con conciencia de sí en diferentes tiempos, en distintos lugares ${ }^{22}$, esto se afirma con Kant para quien el ser humano es una sola y misma persona, en todo momento, aún cuando presenta alteraciones (vida, muerte, nacimiento y enfermedad), por lo que al seguir siendo persona su identidad no cambia, por lo cuál debe ser respetado y tomado en cuenta en la experiencia del cuidado; esto queda de manifiesto cuando la persona por alguna enfermedad neurológica queda sin alguna característica ligada a ella, como la capacidad de razonar, sin embargo en respeto a la identidad que fue, debe ser tratada como tal.

Otro aporte en las características de la persona, se retoma con Kierkegaard y Scheler en el sentido de ser poseedor de valores los que le dan libertad de aprender y actuar con base en ello. Las personas se hacen al hacer efectivos los valores ${ }^{23}$, además de que el tener sentimiento de valor y un saber de esencia es lo que conforma el espíritu racional, ello es lo que distingue a las personas de los animales. Kierkegaard también dice que ante los dilemas (enfermedad), el hombre se libera del mundo y se encuentra en Dios.

La persona es individual, pero es innegable que es un ser social, influido por la cultura en la que se desarrolla y ello le permite vivenciar de determinada forma, la experiencia en la salud y enfermedad, esas situaciones nos deben hacer reflexionar Al momento de vivir la experiencia del cuidado y no ignorarlo, ya que es un gran reto para la enfermera el conocer los componentes de la persona el revalorar a quien cuida y de sí mismo, ya que sólo así estaremos evolucionando como disciplina, a través del estudio y la investigación.

\section{CONCLUSIÓN}

El análisis del concepto de persona, desde una perspectiva filosófica aporta a la disciplina de Enfermería profundización en el conocimiento del ser a quien se brinda atención, ya que es punto de partida para otorgar cuidados en la experiencia de la salud, con conocimiento de aquellas características que no deben ser obviadas al ser cuidador del otro.

En la persona se sintetizan naturaleza racional, cuerpo, alma o espíritu, características que conforman la individualidad de cada ser, que ofrecen la posibilidad de darse cuenta de lo que desea y la conciencia de sí mismo, es lo que le hace elegir aquello que le aportará beneficio en su deseo de alcanzar el bienestar, es lo que le da libertad, aunque esa autonomía en la persona esta influenciada por los valores que rigen su conducta en la sociedad. El reto es grande, pero al cuidar del otro en función de lograr su bienestar implica, tomar en cuenta todo lo que conforma a la persona, en su sentido individual como social, ya que sólo así es posible decir que se cuida del otro, de su existencia o de su fin digno, recordando y respetando la persona que ha existido en el mundo.

Se debe reflexionar en la visión que ha predominado en nuestra profesión sobre la persona, para avanzar hacia otros paradigmas que permitan trascender más allá de la perspectiva técnico - científica y reflexionar en el cuidado, a partir de la comprensión existencial de la persona en su dimensión humana, una de las propuestas para ello está en el estudiar las ciencias humanas a través del paradigma de la fenomenología que las ha enriquecido.

Es imprescindible pues, que se estudie de manera diferente a la persona, para que lo llamado como cuidado responda realmente a ello, es decir construir una asistencia para cada persona y no ajustar a la "persona" a nuestro "cuidado".

Sea entonces éste ensayo un espacio de reflexión en donde se evidencie que la forma de comprender a la persona, implica una forma de vivir y desarrollar el cuidado, en donde los profesionales de enfermería somos absolutamente responsables.

\section{REFERENCIAS BIBLIOGRÁFICAS}

1 Marriner T. A, Raile A. M. Introducción a la teoría de la enfermería En: Historia, importancia y análisis. Modelos y Teorías en Enfermería. España: Elsevier; 2006. pp 3 - 15

2 Vázquez M. L. Aproximaciones a la creación de competencias culturales para el cuidado de la vida. Invest. Educ. Enferm. (on line) 2006; (Consultado 16 abril 2008) (24)2: 136 - 142 Disponible en: http://www.scielo.org.co/scielo. php?script =sci_abstract\&pid $=$ S0120-5307200600020001 $5 \& \operatorname{lng}=$ es\&nrm $=$ iso 
3 Siles G. J, Solano R. C. Estructuras sociales, división sexual del trabajo y enfoques metodológicos. La estructura familiar y la función socio-sanitaria de la mujer. Invest. Educ. Enferm. (on line) 2007; (Consultado 15 abril 2008) (25)1: 66 - 73 Disponible en: http://www.scielo.org.co/ scielo. php?script $=$ sci_arttext\&pid $=$ S0120-53072007000 $100007 \&$ Ing $=$ pt\&nrm=iso. ISSN 0120-5307.

4 Lavalle U. M. Capítulo II. La mujer mexicana a través de los años. Repercusiones históricas y laborales como factores de empuje en la migración internacional. (on line) (consultado 04 mayo 08) Disponible en: http://catarina. udlap.mx/u_dl_a/tales/documentos/ri/rojas_g_i/capitulo2. pdf

5 Guerra F. La medicina precolombina. España. Instituto de Cooperación Iberoamericana; 1990. p 172

6 Sahagún F. B. Capítulo XXXV. De las pláticas que hacían cuando bautizaban la criatura, y del convite que hacian a los niños cuando les ponían el nombre; y de la plática que los viejos hacían a la criatura y a la madre En: Creencias y costumbres. México. Fondo de cultura económica; 1997. p 74

7 Gallegos M. J. Una joya preciosa: Significado del cuidado del niño en México. Texto contexto Enferm, Florianópolis. (on line) 2006; (Consultado 15 abril 2008) 15 (Esp): 146 - 51. Disponible en: http://redalyc.uaemex. $\mathrm{mx} / \mathrm{redalyc} / \mathrm{src} /$ inicio/ArtPdfRed.jsp?iCve $=71409917$.

8 Aguirre B. G. Medicina indígena En: Medicina y Magia. El proceso de enculturación en la estructura colonial. México Instituto Nacional Indigenista; 1963 p 37

9 Quintero L. C. Enfermería en el mundo cristiano. Octubre año/vol. 1número 001. Universidad de la Sabana Chía Colombia AQUICHAN: (on line) 42 (Consultado 17 abril 08)- 47. Disponible en: http://redalyc.uaemex.mx/redalyc/ pdf/741/74110112.pdf

10 Tarrio S. E, Representaciones sociales, ciencia e ideología en el cuidado Enfermero de ancianos dependientes institucionalizados. Universidad Nacional de Mar de Plata. Buenos Aires Argentina (consultado 14 marzo 2008). Disponible en: http:// www.psicologiacientifica.com/bu/ imprimir-79-representaciones-sociales-ciencia-e-ideologiaen-el-cuidado-enfermero-de-ancianos-dependientes-institucionalizados.html

11 Kérouac, S. et al. Grandes Corrientes del Pensamiento En: Pensamiento Enfermero. España. Masson; 1996 pp 1 - 18

12 García M., Caro C. Historia de la Enfermería. Evolución Histórica del cuidado Enfermero. Harcourt. España; 2001 p. 147
13 Fernández del C. F. La Escuela de Enfermeras En: El Hospital General de México. Antecedentes y evolución. México. Talleres Gráficos de la Cia. Editora y Librera ARS S. A; 1946 pp 47 - 50

14 Granda B. P. Cien años de la creación de la Escuela de Enfermería del Hospital General de México Disponible en: http://www.hospitalgeneral.salud.gob.mx/descargas/pdf/ enfermeria/arti_26.pdf consultado 060508

15 Instituto mexicano del seguro social. Historia de la Enfermería (on line) (Consulta 16 abril 2008) Disponible en: http://www.imss.gob.mx/IMSS/IMSS_SITIOS/IMSS_06/ProfesionalesSalud/DPM/Enfermeria/Trayectoria.htm

16 Poblete T. M, Valenzuela S. Cuidado humanizado un desafio para las enfermeras en los servicios hospitalarios. Acta Paul. Enferme. (on line). (Consultado 17 abril 2008). 2007; 20 (4):499 - 503 Disponible en: http://www.scielo.br/pdf/ ape/v20n4/18.pdf

17 Barbosa P. L, Azevedo S. S. Significados y percepciones sobre el Cuidado de Enfermería en la Unidad de Cuidados Intensivos. Index Enferm. (on line) (citado 2008 Marzo 13]. 2006 Nov; 15(54): 20-24. Disponíble en: http:// scielo.isciii.es/scielo.php?script=sci_arttext\&pid $=$ S1132$12962006000200004 \&$ lng $=$ pt\&nrm $=$ iso.

18 De la Cuesta C. El cuidado del otro: desafíos y posibilidades. Invest Educ Enferm. (on line). (Consultado 16 abril 2008); 2007 (25)1: 106-112 Disponible en: http://www. scielo.org.co/scielo.php?script $=$ sci_arttext\&pid $=$ S0120$53072007000100012 \&$ lng $=$ pt\&nrm $=$ iso

19 ibidem

20 Abbagnano Nicola. Concepto de Persona en: Diccionario de Filosofía. México. Fondo de Cultura Económica; 2007. Pp 909 - 912

21 Hirschberger J. Historia de la Filosofía II. Edad moderna. Edad contemporánea. España. Herder. 15ª Ed; 2000. p 100.

22 Rodríguez Y. E. La persona humana, algunas consideraciones. ARS. Médica. Revista de Estudios Médico Humanísticos. Universidad Católica de Chile (on line). (consultado 1404 2008). Disponible en: http://escuela.med.puc. cl/publ/arsmedica/arsmedica6/art11.html 23 Ibidem p. 325, 402.

\section{DIRECCIÓN PARA CORRESPONDENCIA}

Lic. Laura Jiménez:Trujanolaujim_2@yahoo.com.mx 


\section{Lineamientos para la aceptación de artículos en la revista Enfermería Universitaria}

La Revista Enfermería Universitaria es una publicación trimestral de la Escuela Nacional de Enfermería y Obstetricia de la UNAM, su objetivo es difundir las aportaciones de Enfermería en materia de investigación, docencia y práctica profesional, con el propósito de contribuir al fortalecimiento, discusión, e intercambio disciplinar con Instituciones académicas, de salud nacionales e internacionales. La revista a través de su comité editorial y revisor realizan el arbitraje de los escritos propuestos para su publicación como: resultados de investigaciones de Enfermería, docencia, ensayos, experiencias de la práctica profesional.

Los artículos deberán enviarse a Revista Enfermería Universitaria, Camino Antiguo a Xochimilco S/N y Viaducto Tlalpan, Col. San Lorenzo Huipulco, C.P. 14370, México D.F., Fax.: 55730698 ext. 273 o por correo electrónico: revista@eneo.unam.mx

\section{Preparación del manuscrito}

Impresión original y dos copias a doble espacio en papel bond tamaño carta $(21 \times 28 \mathrm{~cm})$ en dos columnas, letra tipo arial 12, con paginación inferior lateral derecha empezando con la carátula.

Grabación en disco flexible (CDRW) con nombres de o de los archivos y formato

Cada parte del escrito en hojas separadas: (carátula, resúmenes y palabras clave, texto del artículo, referencias, cuadros, graficas.

Formato de sesión de derechos de autor a nombre de Revista Enfermería Universitaria. Escuela Nacional de Enfermería y Obstetricia. División de Estudios de Posgrado: Camino Antiguo a Xochimilco S/N y Viaducto Tlalpan, Col. San Lorenzo Huipulco, C.P. 14370, Mexico.D.F

\section{Titulo y Autoría}

Título completo en español y en ingles con extensión de diez a quince palabras que refleje en forma específica y clara el contenido, sin abreviaturas o siglas

Titulo corto en español y en inglés con extensión máxima de ocho palabras

Nombre completo de cada autor, rango académico, centro de trabajo departamento o institución.

Nombre completo y domicilio, teléfono, fax y correo electrónico del autor a quien deberá dirigirse la correspondencia

\section{Resumen y palabras clave}

Cada artículo se acompañara de un resumen en español y en inglés en hojas separadas con una extensión de 150 a 200 palabras que indique claramente el orden de la información en el texto, en el caso de artículos científicos señalar: introducción, objetivo, metodología, resultados, discusión y conclusiones.

Las palabras clave en español y en inglés(Key Words), de tres a seis que permitan identificar el manuscrito.

\section{Cuerpo del Trabajo}

Para artículos de investigación, máximo 20 cuartillas, organizado en introducción, metodología, resultados, discusión, conclusiones y referencias bibliográficas.

Las gráficas y figuras en el orden en que se citan en el trabajo, en hojas separadas y en el caso de utilizar gráficas estas deberán contar con alta resolución; preferentemente sin que sean archivos nativos de Power Point.

\section{Presentación del texto}

a) Estará organizado en las siguientes secciones: 
- Introducción: Incluir el propósito y justificación del trabajo con fundamentos teórico-conceptuales.

- Metodología: En esta sección incluir la descripción de la población de estudio, criterios de selección, tamaño de muestra, tipo de muestro y pérdida de sujetos. En el caso de los estudios clínicos además incluir definición, frecuencia, gravedad y características clínicas de la enfermedad, así como las posibles complicaciones de la intervención. Descripción en forma clara y precisa los métodos, aparatos, procedimientos, medicamentos o sustancias químicas utilizadas, señalando las ventajas, desventajas o limitaciones de uso. Cuando se trata de experimentos con seres humanos, precisar si los procedimientos empleados han respetado los criterios éticos (Declaración de Helsinki). Describir en forma clara y precisa la medición y operacionalización de variables, métodos estadísticos utilizados y plan de análisis, permitiendo así la verificación de los resultados. Presentar las pruebas de validez y confiabilidad, de los instrumentos de medición, definir los términos estadísticos, abreviaturas y los símbolos e indicar los programas informáticos utilizados.

- Resultados: Presentarlos con secuencia lógica en el texto. Enfatizar o resumir sólo los hallazgos relevantes y no repetir en el texto los datos de los cuadros, figuras e ilustraciones. Cuidar que el orden y secuencia de éstos sea el correspondiente a la presentación de los mismos.

- Discusión y conclusiones: Se derivan del análisis de los resultados y propósitos del estudio, destacar los aspectos nuevos o relevantes, así como la explicación a los hallazgos encontrados. Comparar los resultados con otros estudios similares, vincular las conclusiones con los objetivos de estudio y cómo contribuye al campo del conocimiento de Enfermería. Las afirmaciones deben estar plenamente respaldadas por los datos mostrados, además mencionar las limitaciones del estudio.

b) Manuscritos de experiencia de la práctica clínica, extensión promedio de 10 a15 cuartillas. En este caso se estructura como un ensayo con las siguientes secciones:
- Introducción: Identifica los conceptos centrales del tema en estudio, presenta revisión crítica y analítica de la literatura, en congruencia con el tema abordado, justifica la razón por la que se realizó el trabajo y expone los propósitos, refiere la contribución del tema, al conocimiento y práctica de enfermería.

- Desarrollo: Expone las ideas principales del tema, precisa método o técnica, instrumentos, aparatos y procedimientos utilizados (si el tema lo amerita); si es un caso clínico o proceso de enfermería, contiene la información relevante sobre el caso, formula los diagnósticos de enfermería y los problemas interdependientes, refiere las intervenciones de enfermería pertinentes con éstos. Evalúa el cambio de la respuesta humana y el problema interdependiente.

- Conclusiones: Derivan del análisis del trabajo y están relacionadas con el propósito del estudio. Señala las contribuciones del tema abordado al ejercicio profesional de Enfermería. Formula nuevos argumentos o tesis de trabajo y desarrolla los elementos teórico-prácticos que permiten operacionalizar procedimientos.

c) Editorial: Extensión máxima de ocho cuartillas. La estructura de este tipo de documentos es argumentativa, es decir, iniciar con la definición del tema, texto, suceso o evento a tratar; el autor expone su postura, justifica con argumentos su opinión personal y cierra o concluye con comentarios críticos reafirmando la posición adoptada.

\section{Cuadros y Figuras}

Al diseñar cuadros (tablas) y figuras (dibujos gráficos y fotografías) tener en cuenta una posible reducción de tamaño. Los encabezados de cuadros y las leyendas de figura deberán entenderse sin hacer referencia al texto

Los pies de figura están señalados con los números arábigos conforme a la secuencia global que les corresponde. En una hoja separada, se presenta el titulo y la explicación de todas las figuras.

\section{Referencias Bibliográficas}

Todas las referencias bibliográficas deben citarse en el texto con los números consecutivos arábigos en superíndice, según el orden de aparición de las citas, se agregarán al final del artículo y se ajustarán a las normas siguientes: 
Los apellidos paterno y materno de los autores aparecen enlazados con guión corto e inicial del nombre. Ejemplo:

\section{Libros.}

Balseiro-Almario L. Principios de Administración. $2^{\mathrm{a}} \mathrm{Ed}$. Aztlán. México, 1996. 186 pp.

\section{Revistas}

Para el caso de artículos consultados en revistas científicas las normas son las siguientes:

Apellido (s) e inicial (es) del nombre de los autores, seguido (s) del signo coma. En el caso de que los autores sean más de 6 se anotará y Cols. Título completo del trabajo, proposición en acuerdo al Index Medicus, año de publicación punto y coma; número de volúmenes; entre paréntesis número o mes y año del ejemplar, seguido de dos puntos y número de páginas.

\section{Ejemplo:}

Barraza KL, Villareal AJ, Seipp C, Lara P, et al. Prevalecencia de asma y otras enfermedades alérgicas en niños escolares. Salud Pública de México, año 2005, 43(3), 433-477.

\section{Artículos de internet}

Ejemplo: IMERSO. Las personas mayores en España, Informe 2002 (consultado 28/07/2006 on line). Disponible en: www.imerso mayores.csic.es/estadísticas/informacion/informe2002.

\section{Capítulos de libros}

Ejemplo: Jack MQ. Cuidados intensivos en pacientes neurológicos. En: Marino ANET, editors. Cuidados Intensivos Médicos. México: Interamericana Mc Graw-Hill; 1998.p.351-366

\section{Políticas de Publicación}

Las opiniones expresadas por los autores son de su responsabilidad y no reflejan los criterios o políticas de la institución y la Revista.

La recepción del trabajo por la Revista no implica la obligación de su publicación, ni compromiso con respecto a la fecha exacta de su aparición.

No se devolverá ningún artículo sometido a revisión independientemente de que se publique o no.

\section{LOS ARTÍCULOS QUE SE ENVÍEN DEBEN SER ORIGINALES E INÉDITOS.}

La Revista se reserva el derecho legal de reproducción de los artículos.

Los autores principales son los responsables del trabajo y por lo tanto, conservan los créditos del mismo.

\section{Material digital}

Las fotografías incluídas para illustrar el artículo deben contar con tamaño real, estas tienen que ser enviadas de preferencia a la mayor resolución posible y sin adicionarlas en power point o word. Deberán ser anexadas las fotos a un CD de manera individual para que se puedan manipular en los programas de edición fotográfica y con las siguientes extenciones:

- JPG, JPEG, TIFF y EPS.

Importante: Ninguna gráfica, illustración, fotografía o logotipo bajado de la internet tiene los permisos necesarios para su impresión además de no contar con calidad óptima de reproducción.

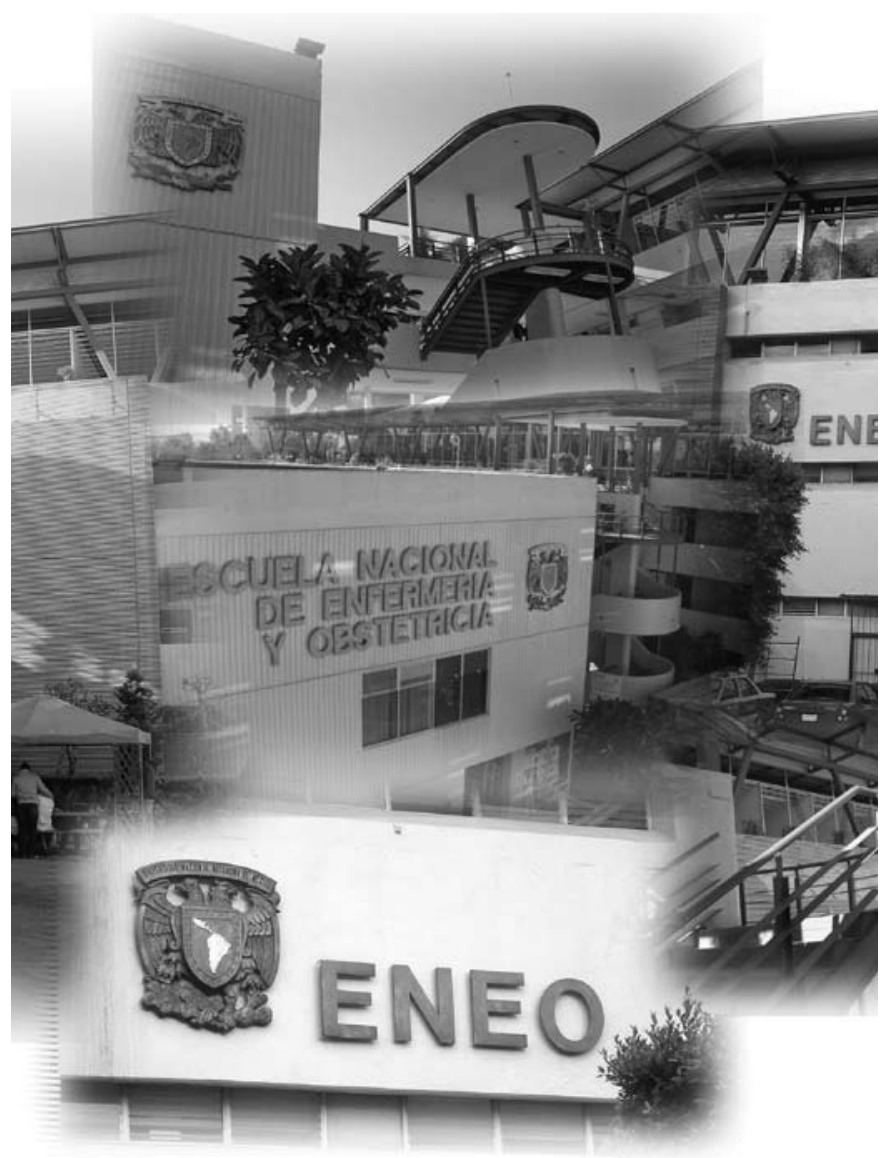




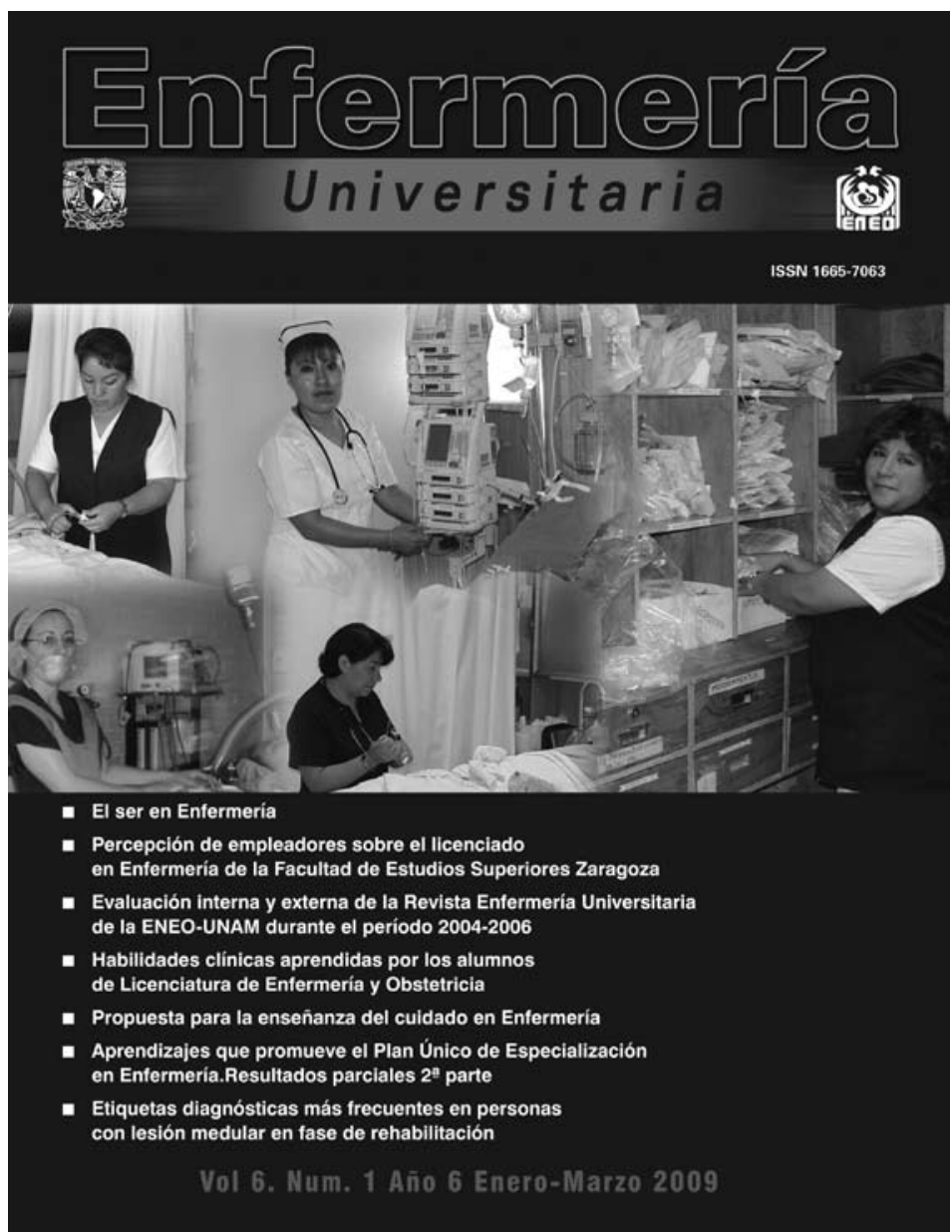

\section{REVISTA ENFERMERIA UNIVERSITARIA}

Escuela Nacional de Enfermería y Obstetricia

Camino Viejo a Xochimilco s/n y Viaducto Tlalpan. Col. San Lorenzo Huipulco, C.P. 14370, México, D.F.

\section{FORMATO PARA LA AUTORIZACION DE PUBLICACION}

Por medio de la presente transferimos a la revista Enfermería Universitaria los derechos del escrito cuyo titulo se indica, incluyendo: 1) derechos para reproducir el articulo completo o partes del mismo, 2) derechos para reimprimir sobretiros del articulo para una venta o distribución gratuita, 3) derechos para reproducir el articulo en una colección de trabajos o en cualquier otro sistema mecánico o electrónico; sin embargo conservamos el derecho para utilizar el material en forma personal para su presentación en conferencias, reuniones de Eenfermería y /o exposiciones científicas.

Firmas de autorización

Firmas de autorización

Firmas de autorización 


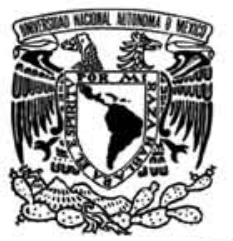

Coordinación de Investigación y DEPARTAMENTO DE SALUD PÚBLICA

Cómo evitar el contagio de la influenza

ila salud está en tus manos:

iEvita contagiar a los demás!

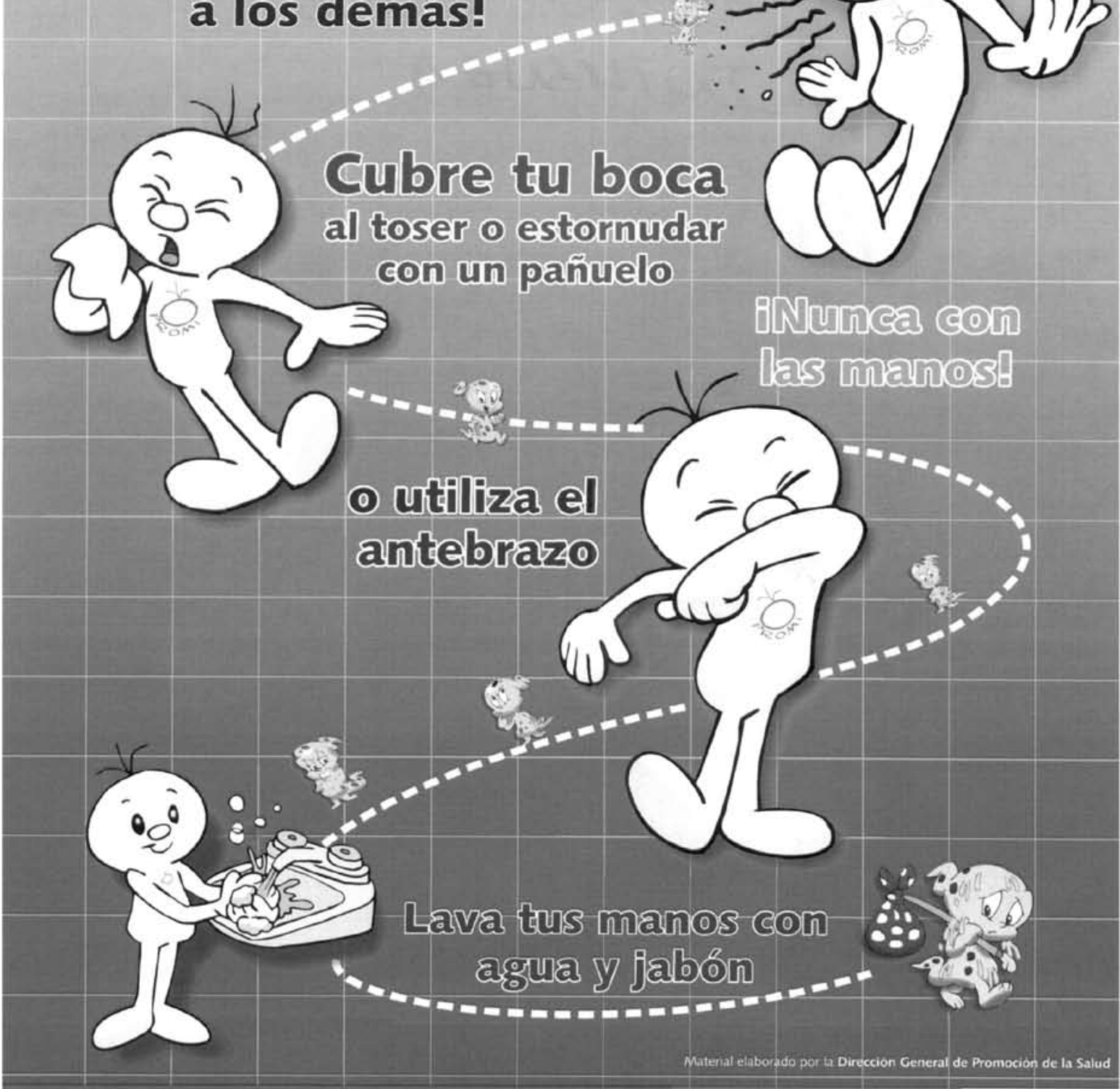

